\title{
Generalized associahedra via brick polytopes
}

\author{
Vincent Pilaud ${ }^{1}$ | and Christian Stump 非 \\ ${ }^{1}$ CNRS \& LIX, École Polytechnique, Palaiseau, France \\ ${ }^{2}$ Institut für Algebra, Zahlentheorie, Diskrete Mathematik, Universität Hannover, Germany
}

\begin{abstract}
We generalize the brick polytope of V. Pilaud and F. Santos to spherical subword complexes for finite Coxeter groups. This construction provides polytopal realizations for a certain class of subword complexes containing all cluster complexes of finite types. For the latter, the brick polytopes turn out to coincide with the known realizations of generalized associahedra, thus opening new perspectives on these constructions. This new approach yields in particular the vertex description and a relevant Minkowski sum decomposition of generalized associahedra.

Résumé. Nous généralisons le polytope des briques de V. Pilaud et F. Santos aux complexes de sous-mots sphériques pour des groupes de Coxeter finis. Cette construction fournit des réalisations polytopales pour une certaine classe de complexes de sous-mots qui contient tous les complexes d'amas de type fini. Pour ces derniers, les polytopes des briques s'avèrent coïncider avec les réalisations connues des associaèdres généralisés, ouvrant ainsi de nouvelles perspectives sur ces constructions. Cette nouvelle approche fournit en particulier la description des sommets et une décomposition pertinente en somme de Minkowski des associaèdres généralisés.
\end{abstract}

Keywords: Coxeter-Catalan combinatorics, subword complexes, cluster complexes, generalized associahedra, Cambrian lattices, Cambrian fans

\section{Introduction}

In [PP12], the first author and M. Pocchiola studied pseudoline arrangements on sorting networks as combinatorial models for triangulations of convex polygons, pseudotriangulations, and multitriangulations. Motivated by the polytopality of the multiassociahedron, the first author and F. Santos [PS12a] defined the brick polytope of a sorting network. This construction provides polytopal realizations of the simplicial complexes associated to a certain class of sorting networks. In particular, it turns out that all type $A$ associahedra of C. Hohlweg and C. Lange [HL07] appear as brick polytopes for well-chosen sorting networks.

Independently in [Stu11], the second author connected multitriangulations and subword complexes of type $A$, which can be visually interpreted with sorting networks (see also a generalization developed in [SS12]). Subword complexes on Coxeter groups were defined by A. Knutson and E. Miller [KM04, KM05] in the context of Gröbner geometry of Schubert varieties. They proved that these simplicial complexes are either topological balls or spheres, and raised the question of realizing spherical subword complexes as boundary complexes of convex polytopes.

\footnotetext{
†Partially supported by grant MTM2008-04699-C03-02 and MTM2011-22792 of the spanish Ministerio de Ciencia e Innovación, by European Research Project ExploreMaps (ERC StG 208471), and by a postdoctoral grant of the Fields Institute of Toronto.

$\ddagger$ Partially supported by a CRM-ISM fellowship, and by the DFG via the Research Group "Methods for Discrete Structures". 
Recently, C. Ceballos, J.-P. Labbé, and the second author [CLS11] extended the subword complex interpretations of [Stu11] and [PP12] for triangulations and multitriangulations of convex polygons to general finite types. They proved in particular that all finite type cluster complexes of S. Fomin and A. Zelevinsky [FZ03] are isomorphic to certain well-chosen subword complexes. The first polytopal realizations of cluster complexes of finite types were obtained by F. Chapoton, S. Fomin and A. Zelevinsky [CFZ02]. Later, C. Hohlweg, C. Lange and H. Thomas [HLT11] extended the ideas of [HL07] to construct different realizations based on the Cambrian fan studied by N. Reading and D. Speyer [Rea04, Rea06, Rea07, RS09]. In his recent preprint [Ste11], S. Stella generalizes the approach of [CFZ02] and shows that the resulting realizations of the associahedra coincide with that of [HLT11].

In this paper, we define the brick polytope of any subword complex for a finite Coxeter group (see Section 47. As in type $A$, the brick polytope does not realize its subword complex in general. We study the class $\mathfrak{R}$ of subword complexes which are realized by their brick polytope. They are simply characterized in terms of their root configuration (see Section 3). The class $\mathfrak{R}$ contains in particular the finite type cluster complexes. For these, the brick polytopes turn out to be (translates of) the realizations of the generalized associahedra described in [CFZ02, HLT11, Ste11] (see Section 6). We therefore obtain the vertex description of these realizations and decompose them into Minkowski sums of Coxeter matroid polytopes as defined in [BGW97]. Furthermore, we extend relevant combinatorial properties of these associahedra to any subword complex of the class $\mathfrak{R}$ (see Section 5). We relate the normal fan of the brick polytope to the Coxeter fan, and the graph of the brick polytope to a quotient of the weak order.

In the classical situation of type $A$, our definition of the brick polytope matches that of [PS12a]. This situation provides relevant examples, which are moreover visually accessible using sorting networks. We refer to the long version of this paper [PS12b] for type $A$ examples as well as additional illustrations and pictures, which have been omitted in this extended abstract for space reasons.

\section{Background on Coxeter groups and subword complexes}

\subsection{Finite Coxeter groups}

We recall classical notions on finite Coxeter groups. We refer to [Hum90] for more details.

Coxeter system. Let $(V,\langle\cdot \mid \cdot\rangle)$ be an $n$-dimensional euclidean vector space. For any vector $v \in V \backslash 0$, we denote by $s_{v}$ the reflection interchanging $v$ and $-v$ while fixing the orthogonal hyperplane pointwise. We consider a finite Coxeter group $W$ acting on $V$, that is, a finite group generated by reflections. The Coxeter arrangement of $W$ is the collection of all reflecting hyperplanes. Its complement in $V$ is a union of open polyhedral cones. Their closures are called chambers. The Coxeter fan is the polyhedral fan formed by the chambers together with all their faces. This fan is complete (its cones cover $V$ ) and simplicial (all cones are simplicial), and we can assume without loss of generality that it is essential (the intersection of all chambers is reduced to the origin). We fix an arbitrary chamber $\mathcal{C}$ which we call the fundamental chamber. The simple reflections of $W$ are the $n$ reflections orthogonal to the facet defining hyperplanes of $\mathcal{C}$. The set $S$ of simple reflections generates $W$. The couple $(W, S)$ is a Coxeter system.

Roots and weights. For simple reflections $s, t \in S$, denote by $m_{s t}$ the order of the product $s t \in W$. We fix a generalized Cartan matrix for $(W, S)$, i.e. a matrix $\left[a_{s t}\right]_{s, t \in S}$ such that $a_{s s}=2, a_{s t} \leq 0$, $a_{s t} a_{t s}=4 \cos ^{2}\left(\frac{\pi}{m_{s t}}\right)$ and $a_{s t}=0 \Leftrightarrow a_{t s}=0$ for all $s, t \in S$. We can associate to each simple reflection $s$ a simple root $\alpha_{s} \in V$, orthogonal to the reflecting hyperplane of $s$ and pointing toward the half-space containing $\mathcal{C}$, in such a way that $s\left(\alpha_{t}\right)=\alpha_{t}-a_{s t} \alpha_{s}$ for all $s, t \in S$. The set of all simple roots 
is denoted by $\Delta:=\left\{\alpha_{s} \mid s \in S\right\}$. The orbit $\Phi:=\left\{w\left(\alpha_{s}\right) \mid w \in W, s \in S\right\}$ of $\Delta$ under $W$ is a root system for $W$. It is clearly invariant under the action of $W$ and contains precisely two opposite roots orthogonal to each reflecting hyperplane of $W$.

The set $\Delta$ of simple roots forms a linear basis of $V$ (since we assumed $W$ to act essentially on $V$ ). The root system $\Phi$ is the disjoint union of the positive roots $\Phi^{+}:=\Phi \cap \mathbb{R}_{\geq 0}[\Delta]$ (non-negative linear combinations of the simple roots) and the negative roots $\Phi^{-}:=-\Phi^{+}$. In other words, the positive roots are the roots whose scalar product with any vector of the interior of the fundamental chamber $\mathcal{C}$ is positive, and the simple roots form the basis of the cone generated by $\Phi^{+}$.

The set of fundamental weights $\nabla:=\left\{\omega_{s} \mid s \in S\right\}$ of $(W, S)$ is the dual basis to the renormalized root basis $\left\{2 \alpha_{s} /\left\langle\alpha_{s} \mid \alpha_{s}\right\rangle \mid s \in S\right\}$. In other words, the weights are defined by $\left\langle\alpha_{s} \mid \omega_{t}\right\rangle=\delta_{s=t}\left\langle\alpha_{s} \mid \alpha_{s}\right\rangle / 2$ for all $s, t \in S$. Thus, $W$ acts on the weight basis by $s\left(\omega_{t}\right)=\omega_{t}-\delta_{s=t} \alpha_{s}$. Geometrically, the weight $\omega_{s}$ gives the direction of the ray of the fundamental chamber $\mathcal{C}$ not contained in the reflecting hyperplane of $s$.

Coxeter permutahedra. The $W$-permutahedron $\mathcal{P}_{q}(W)$ is the convex hull of the orbit under $W$ of a point $q$ in the interior of the fundamental chamber $\mathcal{C}$ of $W$. Its normal fan is the Coxeter fan of $W$. If $q=\sum_{s \in S} \omega_{s}$, then $\mathcal{P}_{q}(W)$ is (a translate of) the Minkowski sum of all positive roots (each considered as a one-dimensional polytope). We call it the balanced $W$-permutahedron, and denote it by $\mathcal{P}(W)$.

Words on $S$. The length $\ell(w)$ of an element $w \in W$ is the length of the smallest expression of $w$ as a product of the generators in $S$. An expression $w=w_{1} \cdots w_{p}$ with $w_{1}, \ldots, w_{p} \in S$ is called reduced if $p=\ell(w)$. We denote by $w_{\mathrm{o}}$ the unique longest element in $W$. The (right) weak order on $W$ is the partial order $\leq$ defined by $u \leq w$ if there exists $v \in W$ such that $u v=w$ and $\ell(u)+\ell(v)=\ell(w)$. It defines a lattice structure on the elements of $W$ with minimal element being the identity $e \in W$ and with maximal element being $w_{\circ}$. Its Hasse diagram is the graph of the $W$-permutahedron (oriented from $e$ to $w_{\circ}$ ).

We denote by $S^{*}$ the set of words on the alphabet $S$, and by $\varepsilon$ the empty word. To distinguish them, we denote words in $S^{*}$ with square letters like w $\in S^{*}$ and group elements with normal letters like $w \in W$. The Demazure product on the Coxeter system $(W, S)$ is the function $\delta: S^{*} \rightarrow W$ defined inductively by

$$
\delta(\varepsilon)=e \quad \text { and } \quad \delta(\mathrm{Qs})= \begin{cases}\delta(\mathrm{Q}) s & \text { if } \ell(\delta(\mathrm{Q}) s)=\ell(\delta(\mathrm{Q}))+1 \\ \delta(\mathrm{Q}) & \text { if } \ell(\delta(\mathrm{Q}) s)=\ell(\delta(\mathrm{Q}))-1\end{cases}
$$

\subsection{Subword complexes}

Let $(W, S)$ be a Coxeter system, let $\mathrm{Q}:=\mathrm{q}_{1} \mathrm{q}_{2} \cdots \mathrm{q}_{m} \in S^{*}$ and let $\rho \in W$. In [KM04, KM05], A. Knutson and E. Miller define the subword complex $\mathcal{S}(\mathrm{Q}, \rho)$ as the pure simplicial complex of subwords of $\mathrm{Q}$ whose complements contain a reduced expression of $\rho$. The vertices of this simplicial complex are labeled by (positions of) the letters in the word Q. Note that two positions are different even if the letters of $Q$ at these positions coincide. We denote by $[m]:=\{1, \ldots, m\}$ the set of positions in $Q$. The facets of the subword complex $\mathcal{S}(\mathrm{Q}, \rho)$ are the complements of the reduced expressions of $\rho$ in the word $\mathrm{Q}$.

It is proved in [KM04] that the subword complex $\mathcal{S}(\mathrm{Q}, \rho)$ is a sphere if $\rho=\delta(\mathrm{Q})$, and a ball otherwise. In this paper we only consider spherical subword complexes. By appending to the word $\mathrm{Q}$ any reduced expression of $\delta(\mathrm{Q})^{-1} w_{\circ}$, we obtain a word $\mathrm{Q}^{\prime}$ whose Demazure product is $\delta\left(\mathrm{Q}^{\prime}\right)=w_{\circ}$ and such that the spherical subword complexes $\mathcal{S}(\mathrm{Q}, \delta(\mathrm{Q}))$ and $\mathcal{S}\left(\mathrm{Q}^{\prime}, w_{\circ}\right)$ are isomorphic (see [CLS11, Theorem 3.7] for details). Consequently, we furthermore assume that $\rho=\delta(\mathrm{Q})=w_{\circ}$ to simplify the presentation without loss of generality, and we write $\mathcal{S}(\mathrm{Q})$ instead of $\mathcal{S}\left(\mathrm{Q}, w_{\circ}\right)$ to simplify notations.

For any facet $I$ of $\mathcal{S}(\mathrm{Q})$ and any element $i \in I$, there is a unique facet $J$ of $\mathcal{S}(\mathrm{Q})$ and an element $j \in J$ such that $I \backslash i=J \backslash j$. We say that $I$ and $J$ are adjacent facets, and that $J$ is obtained from $I$ by flipping $i$. 


\section{Root configurations}

Definition 3.1 To a facet I of $\mathcal{S}(\mathrm{Q})$ and a position $k \in[m]$, we associate a root $\mathrm{r}(I, k):=\sigma_{I}^{[1, k-1]}\left(\alpha_{q_{k}}\right)$, where $\sigma_{I}^{[x, y]}$ denotes the product of the reflections $q_{z}$ for $z \in[x, y] \backslash I$ in this order. The root configuration of the facet $I$ is the multiset $\mathrm{R}(I):=\{[\mathrm{r}(I, i) \mid i \in I\}$ of all roots associated to the elements of $I$.

Throughout this paper, we show that the combinatorial and geometric properties of the root configuration $\mathrm{R}(I)$ encode many properties of the facet $I$. We first note that, for a given facet $I$ of $\mathcal{S}(\mathrm{Q})$, the map $\mathrm{r}(I, \cdot):[m] \rightarrow \Phi$ can be used to understand the flips in $I$ (see [CLS11, Section 3.1] for similar statements and proofs).

Lemma 3.2 Let I be any facet of the subword complex $\mathcal{S}(\mathrm{Q})$.

(1) The map $\mathrm{r}(I, \cdot): k \mapsto \mathrm{r}(I, k)$ is a bijection between the complement of $I$ and $\Phi^{+}$.

(2) If I and $J$ are two adjacent facets of $\mathcal{S}(\mathrm{Q})$ with $I \backslash i=J \backslash j$, the position $j$ is the unique position in the complement of I for which $\mathrm{r}(I, i) \in\{ \pm \mathrm{r}(I, j)\}$. Moreover, $\mathrm{r}(I, i)=\mathrm{r}(I, j) \in \Phi^{+}$if $i<j$, while $\mathrm{r}(I, i)=-\mathrm{r}(I, j) \in \Phi^{-}$if $j<i$.

(3) In the situation of 2), the map $\mathrm{r}(J, \cdot)$ is obtained from the map $\mathrm{r}(I, \cdot)$ by:

$$
\mathrm{r}(J, k)= \begin{cases}s_{\mathrm{r}(I, i)}(\mathrm{r}(I, k)) & \text { if } \min (i, j)<k \leq \max (i, j), \\ \mathrm{r}(I, k) & \text { otherwise. }\end{cases}
$$

The geometry of the root configuration $\mathrm{R}(I)$ encodes certain combinatorial properties of $I$. In fact, the facet $I$ is relevant for the brick polytope only when its root configuration is pointed. This is in particular the case when $\mathrm{R}(I)$ forms a basis of $V$. From Lemma 3.2 and the connectedness of the flip graph, we derive that this property depends only on $\mathrm{Q}$, not on the particular facet $I$ of $\mathcal{S}(\mathrm{Q})$.

Lemma 3.3 Either all the root configurations $\mathrm{R}(I)$, for I facet of $\mathcal{S}(\mathrm{Q})$, are simultaneously linear bases of the vector space $V$, or none of them is.

In this paper, we assume that the word $\mathrm{Q}$ is such that the root configuration $\mathrm{R}(I)$ forms a linear basis of $V$ for any facet $I$ of $\mathcal{S}(\mathrm{Q})$. We say that the word Q is realizing, and we denote by $\mathfrak{R}$ the class of realizing words. Note that if the root configuration is linearly independent but does not span $V$, we can always find an isomorphic subword complex for a parabolic subgroup for which the word is indeed realizing (the proof is similar to that of [CLS11, Lemma 5.4]).

Proposition 3.4 Let $\mathrm{Q}$ be a word such that the root configuration $\mathrm{R}(I)$ is linearly independent. Then there exists a word $\mathrm{Q}^{\prime}$ for a parabolic subgroup of $W$ such that $\mathcal{S}(\mathrm{Q})$ and $\mathcal{S}\left(\mathrm{Q}^{\prime}\right)$ are isomorphic and moreover, that $\mathrm{R}\left(I^{\prime}\right)$ forms indeed a linear basis for any facet $I^{\prime}$ of $\mathcal{S}\left(\mathrm{Q}^{\prime}\right)$.

We now consider the geometry of the root configuration $\mathrm{R}(I)$ of a facet $I$ of $\mathcal{S}(\mathrm{Q})$ with respect to a linear functional $f: V \rightarrow \mathbb{R}$. We say that the flip of an element $i$ in $I$ is $f$-preserving if $f(\mathrm{r}(I, i))=0$. We denote by $\mathcal{S}_{f}(\mathrm{Q}):=\{I$ facet of $\mathcal{S}(\mathrm{Q}) \mid \forall i \in I, f(\mathrm{r}(I, i)) \geq 0\}$ the set of facets of $\mathcal{S}(\mathrm{Q})$ whose root configuration is contained in the closed positive halfspace defined by $f$.

Proposition 3.5 The set $\mathcal{S}_{f}(\mathrm{Q})$ forms a connected component of the graph of $f$-preserving flips on $\mathcal{S}(\mathrm{Q})$. 
Proof: Assume that $I$ and $J$ are two adjacent facets of $\mathcal{S}(\mathrm{Q})$ related by an $f$-preserving flip. We write $I \backslash i=J \backslash j$ with $f(\mathrm{r}(I, i))=0$. By Lemma 3.2 3, , we have $\mathrm{r}(J, k)-\mathrm{r}(I, k) \in \mathbb{R} \cdot \mathrm{r}(I, i)$ and thus $f(\mathrm{r}(J, k))=f(\mathrm{r}(I, k))$ for all $k \in[m]$. Thus, $\mathcal{S}_{f}(\mathrm{Q})$ is closed under $f$-preserving flips.

It remains to prove that $\mathcal{S}_{f}(\mathrm{Q})$ is connected by $f$-preserving flips. Let $I$ be a facet of $\mathcal{S}_{f}(\mathrm{Q})$, and define $P:=\{i \in I \mid f(\mathrm{r}(I, i))>0\}$. We prove that we can reconstruct $P$, using an inductive argument on the cardinality of $P$. The connectedness of $\mathcal{S}_{f}(\mathrm{Q})$ then follows from that of the subword complex $\mathcal{S}\left(\mathrm{Q}_{[m] \backslash P}\right)$ of the word obtained from Q by erasing the letters at the positions given by $P$.

Assume first that $P=\{p\}$, and that $\mathrm{r}(I, p) \in \Phi^{-}$. Performing $f$-preserving flips, we can make sure that $\mathrm{r}(I, i) \in \Phi^{-}$for each $i \in I$ with $f(\mathrm{r}(I, i))=0$. Then $j \in I$ iff $\sigma_{I \cap[j-1]}^{[1, j-1]}\left(\alpha_{q_{j}}\right) \in f^{-1}\left(\mathbb{R}_{>0}\right) \cap \Phi^{-}$, and thus the facet $I$ can be reconstructed scanning the word Q from left to right. If $r(I, p) \in \Phi^{+}$, we argue similarly scanning the word $\mathrm{Q}$ from right to left.

Assume now that $P=P^{\prime} \cup\{p\}$, with $P^{\prime} \neq \emptyset$. Consider an auxiliary linear function $f^{\prime}$ which vanishes on $\mathrm{R}(I) \cap f^{-1}(0)$ and on $\mathrm{r}(I, p)$ (this function exists because $\mathrm{R}(I)$ forms a basis of $V$ ). Then $I$ is a facet of $\mathcal{S}_{f^{\prime}}(\mathrm{Q})$, so that we can reconstruct the positions $P^{\prime}$ by induction hypothesis. Finally, since $I \backslash P^{\prime}$ is also a facet of $\mathcal{S}\left(\mathrm{Q}_{[m] \backslash P^{\prime}}\right)$, we can recover the last position $p$ with a similar argument as before.

Corollary 3.6 The set $\mathcal{S}_{f}(\mathrm{Q})$ is a face of the subword complex $\mathcal{S}(\mathrm{Q})$.

Proof: Define again the set of positions $P:=\{k \in[m] \mid f(\mathrm{r}(I, k))>0\}$ for some facet $I$ of $\mathcal{S}_{f}(\mathrm{Q})$. Since the set $\mathcal{S}_{f}(\mathrm{Q})$ is connected by $f$-preserving flips and an $f$-preserving flip preserves $f(\mathrm{r}(I, k))$ for all $k \in[m]$, the set $P$ is independent of the choice of $I \in \mathcal{S}_{f}(\mathrm{Q})$. Furthermore, any position $i \in I \backslash P$ is an $f$-preserving flip. Consequently, $\mathcal{S}_{f}(\mathrm{Q})$ is precisely the set of facets of $\mathcal{S}(\mathrm{Q})$ which contains $P$.

\section{Generalized brick polytopes}

We generalize the definition of brick polytopes of [PS12a] to any spherical subword complex $\mathcal{S}(\mathrm{Q})$.

Definition 4.1 To a facet I of $\mathcal{S}(\mathrm{Q})$ and a position $k \in[m]$, we associate a weight $\mathrm{w}(I, k):=\sigma_{I}^{[1, k-1]}\left(\omega_{q_{k}}\right)$. The brick vector of the facet $I$ is the vector $\mathrm{B}(I):=\sum_{k \in[m]} \mathrm{w}(I, k)$. The brick polytope $\mathcal{B}(\mathrm{Q})$ of the word $\mathrm{Q}$ is the convex hull $\mathcal{B}(\mathrm{Q}):=\operatorname{conv}\{\mathrm{B}(I) \mid$ I facet of $\mathcal{S}(\mathrm{Q})\}$ of all the brick vectors.

The root function $r(\cdot, \cdot)$ and the weight function $\mathrm{w}(\cdot, \cdot)$ have similar definitions and are indeed closely related. The following lemma underlines some similarities (we skip again this straightforward proof).

Lemma 4.2 Let I be a facet of the subword complex $\mathcal{S}(\mathrm{Q})$.

(1) We have $\mathrm{w}(I, k+1)= \begin{cases}\mathrm{w}(I, k) & \text { if } k \in I, \\ \mathrm{w}(I, k)-\mathrm{r}(I, k) & \text { if } k \notin I .\end{cases}$

(2) If I and $J$ are two adjacent facets of $\mathcal{S}(\mathrm{Q})$, with $I \backslash i=J \backslash j$, then $\mathrm{w}(J, \cdot)$ is obtained from $\mathrm{w}(I, \cdot)$ by:

$$
\mathrm{w}(J, k)= \begin{cases}s_{\mathrm{r}(I, i)}(\mathrm{w}(I, k)) & \text { if } \min (i, j)<k \leq \max (i, j), \\ \mathrm{w}(I, k) & \text { otherwise. }\end{cases}
$$

(3) For $j \notin I$, we have $\langle\mathrm{r}(I, j) \mid \mathrm{w}(I, k)\rangle$ is non-negative if $j \geq k$, and non-positive if $j<k$. 
Although we defined brick vectors and brick polytopes for any word Q, we only present here their geometric and combinatorial properties when $\mathrm{Q}$ is realizing. We will see that this condition is sufficient for the brick polytope to realize its subword complex. Reciprocally, the brick polytope can realize its subword complex only when the root configurations of the facets are linearly independent, and we can then assume that the word $\mathrm{Q}$ is realizing by Proposition 3.4. The general situation where $\mathrm{Q} \in S^{*}$ is an arbitrary word with Demazure product $\delta(\mathrm{Q})=w_{\circ}$ is more involved and will be discussed in a subsequent paper.

Lemma 4.3 If $I$ and $J$ are two adjacent facets of $\mathcal{S}(\mathrm{Q})$, with $I \backslash i=J \backslash j$, then the difference of the brick vectors $\mathrm{B}(I)-\mathrm{B}(J)$ is a positive multiple of $\mathrm{r}(I, i)$.

Proof: Since $I$ and $J$ play symmetric roles, we can assume that $i<j$. Then, Lemma 4.2 implies that

$$
\mathrm{B}(I)-\mathrm{B}(J)=\sum_{k \in[m]} \mathrm{w}(I, k)-\mathrm{w}(J, k)=\left(\sum_{i<k \leq j} 2\langle\mathrm{r}(I, j) \mid \mathrm{w}(I, k)\rangle\right) \cdot \mathrm{r}(I, i),
$$

where the last sum is positive since all its summands are non-negative and the last one is positive.

Let $f: V \rightarrow \mathbb{R}$ be a linear functional. We denote by $\mathcal{B}_{f}(\mathrm{Q})$ the face of $\mathcal{B}(\mathrm{Q})$ which maximizes $f$. Remember that $\mathcal{S}_{f}(\mathrm{Q})$ denotes the set of facets of $\mathcal{S}(\mathrm{Q})$ whose root configuration is included in the closed positive halfspace defined by $f$.

Lemma 4.4 The faces of the brick polytope $\mathcal{B}(\mathrm{Q})$ are faces of the subword complex $\mathcal{S}(\mathrm{Q})$. For any facet I of $\mathcal{S}(\mathrm{Q})$, we have $\mathrm{B}(I) \in \mathcal{B}_{f}(\mathrm{Q}) \Longleftrightarrow I \in \mathcal{S}_{f}(\mathrm{Q})$.

Proof: Let $I$ be a facet of $\mathcal{S}(\mathrm{Q})$ such that $\mathrm{B}(I) \in \mathcal{B}_{f}(\mathrm{Q})$. Let $i \in I$, let $J$ be the facet obtained from $I$ by flipping $i$, and let $\lambda>0$ be such that $\mathrm{r}(I, i)=\lambda(\mathrm{B}(I)-\mathrm{B}(J))$ (by Lemma 4.3). Then we have $f(\mathrm{r}(I, i))=\lambda(f(\mathrm{~B}(I))-f(\mathrm{~B}(J))) \geq 0$ since $\mathrm{B}(I)$ maximizes $f$. Thus, $I \in \mathcal{S}_{f}(\mathrm{Q})$. We obtained that the set of facets $I$ of $\mathcal{S}(\mathrm{Q})$ such that $\mathrm{B}(I) \in \mathcal{B}_{f}(\mathrm{Q})$ is contained in $\mathcal{S}_{f}(\mathrm{Q})$. Since the former is closed while the latter is connected under $f$-preserving flips (by Proposition 3.5), they coincide.

Proposition 4.5 For any facet I of $\mathcal{S}(\mathrm{Q})$, the cone of the brick polytope $\mathcal{B}(\mathrm{Q})$ at the brick vector $\mathrm{B}(I)$ coincides with the cone generated by the negative of the root configuration $\mathrm{R}(I)$ of $I$. We set

$$
\mathrm{C}(I):=\text { cone }\{\mathrm{B}(J)-\mathrm{B}(I) \mid J \text { facet of } \mathcal{S}(\mathrm{Q})\}=\text { cone }\{-\mathrm{r}(I, i) \mid i \in I\} .
$$

Proof: Let $\mathrm{C}_{\mathrm{B}}(I)$ be the cone of $\mathcal{B}(\mathrm{Q})$ at $\mathrm{B}(I)$ and let $\mathrm{C}_{\mathrm{R}}(I)$ be the cone generated by $-\mathrm{R}(I)$. The inclusion $\mathrm{C}_{\mathrm{R}}(I) \subset \mathrm{C}_{\mathrm{B}}(I)$ is an immediate consequence of Lemma 4.3 . For the other direction, we need to prove that any face of $\mathrm{C}_{\mathrm{R}}(I)$ is also a face of $\mathrm{C}_{\mathrm{B}}(I)$. That is to say that the brick vector $\mathrm{B}(I)$ maximizes any linear functional $f$ among the brick vectors of all facets of $\mathcal{S}(\mathrm{Q})$ as soon as it maximizes $f$ among the brick vectors of the facets adjacent to $I$. This is ensured by Lemma 4.4

Theorem 4.6 If $\mathrm{Q}$ is realizing, the polar of the brick polytope $\mathcal{B}(\mathrm{Q})$ realizes the subword complex $\mathcal{S}(\mathrm{Q})$.

Proof: Proposition 4.5 ensures that the brick vector of each facet of $\mathcal{S}(\mathrm{Q})$ is a vertex of $\mathcal{B}(\mathrm{Q})$ and that this polytope is full-dimensional and simple. Since the faces of the brick polytope are faces of the subword complex by Lemma 4.4, it immediately implies that the boundary complex of the brick polytope $\mathcal{B}(\mathrm{Q})$ is isomorphic to the subword complex $\mathcal{S}(\mathrm{Q})$.

Remark 4.7 If $(W, S)$ is crystallographic (i.e. stabilizes the lattice $\Lambda$ generated by $\Delta$ ), then the brick polytope $\mathcal{B}(\mathrm{Q})$ is furthermore a lattice polytope (i.e. its vertices are elements of $\Lambda$ ). 


\section{Further combinatorial and geometric properties}

In this section, we consider further relevant properties of the brick polytope $\mathcal{B}(\mathrm{Q})$. We first define an important surjective map $\kappa$ from the Coxeter group $W$ to the facets of the subword complex $\mathcal{S}(\mathrm{Q})$. Using this map, we relate the normal fan of $\mathcal{B}(\mathrm{Q})$ to the Coxeter fan, and the graph of $\mathcal{B}(\mathrm{Q})$ to the weak order. Finally, we decompose $\mathcal{B}(\mathrm{Q})$ into a Minkowski sum of $W$-matroid polytopes. Again, our presentation currently focuses on realizing words, although most of these results seem to extend to non-realizing ones.

\subsection{A surjective map}

A set $U \subset \Phi$ is separable if there is a hyperplane which separates $U$ from its complement $\Phi \backslash U$. For finite Coxeter systems, separable sets are precisely the sets $w\left(\Phi^{+}\right)$, for $w \in W$. According to Proposition 3.5. there is a unique facet $I$ of $\mathcal{S}(\mathrm{Q})$ whose root configuration $\mathrm{R}(I)$ is contained in a given separable set. This defines a map $\kappa$ from $W$ to the facets of $\mathcal{S}(\mathrm{Q})$ which associates to $w \in W$ the unique facet $\kappa(w)$ of $\mathcal{S}(\mathrm{Q})$ such that $\mathrm{R}(\kappa(w)) \subset w\left(\Phi^{+}\right)$. Furthermore, since we assumed that any root configuration forms a basis (and thus is pointed), this map $\kappa$ is surjective. However, note that this map is not injective.

Lemma 5.1 Let $w \in W$ and $\alpha \in \Delta$. If $w(\alpha) \notin \mathrm{R}(\kappa(w))$, then $\kappa\left(w s_{\alpha}\right)$ equals $\kappa(w)$. Otherwise, $\kappa\left(w s_{\alpha}\right)$ is obtained from $\kappa(w)$ by flipping the unique $i \in \kappa(w)$ such that $w(\alpha)=\mathrm{r}(\kappa(w), i)$.

Proof: If $w(\alpha) \notin \mathrm{R}(\kappa(w))$, then $\mathrm{R}(\kappa(w)) \subset w s_{\alpha}\left(\Phi^{+}\right)=w\left(\Phi^{+}\right) \triangle\{ \pm w(\alpha)\}$ (where $\triangle$ denotes the symmetric difference). Thus, $\kappa\left(w s_{\alpha}\right)=\kappa(w)$ by uniqueness in the definition of the map $\kappa$.

Assume now that $w(\alpha)=\mathrm{r}(\kappa(w), i)$ for some $i \in \kappa(w)$, and let $J$ denote the facet of $\mathcal{S}(\mathrm{Q})$ obtained from $\kappa(w)$ by flipping $i$, and $j$ be the position in $J$ but not in $\kappa(w)$. According to Lemma 3.2 2),

$$
\mathrm{r}(J, k)= \begin{cases}s_{w(\alpha)}(\mathrm{r}(\kappa(w), k))=w s_{\alpha} w^{-1}(\mathrm{r}(\kappa(w), k)) & \text { if } \min (i, j)<k \leq \max (i, j), \\ \mathrm{r}(\kappa(w), k) & \text { otherwise. }\end{cases}
$$

If $\min (i, j)<k \leq \max (i, j)$, we have $\mathrm{r}(J, k) \in w s_{\alpha}\left(\Phi^{+}\right)$since $w^{-1}(\mathrm{r}(\kappa(w), k)) \in \Phi^{+}$. Otherwise, $w^{-1}(\mathrm{r}(\kappa(w), k)) \in \Phi^{+} \backslash \alpha$ so that $s_{\alpha} w^{-1}(\mathrm{r}(\kappa(w), k)) \in \Phi^{+}$, and thus $\mathrm{r}(J, k)=\mathrm{r}(\kappa(w), k) \in w s_{\alpha}\left(\Phi^{+}\right)$. We obtain that $\mathrm{R}(J) \subset w s_{\alpha}\left(\Phi^{+}\right)$, and consequently $J=\kappa\left(w s_{\alpha}\right)$ by uniqueness in the definition of $\kappa$.

\subsection{A normal fan description}

Remember that the normal cone of a face $F$ of a polytope $\Pi \subset V$ is the cone of all vectors $v \in V$ such that the linear function $x \mapsto\langle x \mid v\rangle$ on $\Pi$ is maximized by all points in $F$. The normal fan of $\Pi$ is the complete polyhedral fan formed by the normal cones of all faces of $\Pi$. For example, the normal fan of the $W$-permutahedron is the Coxeter fan of $W$. The following proposition relates the normal fan of the brick polytope to the Coxeter fan.

Proposition 5.2 Let I be a facet of $\mathcal{S}(\mathrm{Q})$. The normal cone $\mathrm{C}^{\diamond}(I)$ of $\mathrm{B}(I)$ in $\mathcal{B}(\mathrm{Q})$ is the union of the chambers $w(\mathcal{C})$ of the Coxeter fan of $W$ given by the elements $w \in W$ with $\kappa(w)=I$.

Proof: For a given cone $X \subset V$, we denote by $X^{\diamond}:=\{v \in V \mid \forall x \in X,\langle x \mid v\rangle \geq 0\}$ its polar cone. Then

$$
\mathrm{C}^{\diamond}(I)=\mathrm{C}(I)^{\diamond}=\operatorname{cone}(-\mathrm{R}(I))^{\diamond}=\left(\bigcap_{w \in \kappa^{-1}(I)} w\left(\mathcal{C}^{\diamond}\right)\right)^{\diamond}=\bigcup_{w \in \kappa^{-1}(I)} w\left(\mathcal{C}^{\diamond}\right)^{\diamond}=\bigcup_{w \in \kappa^{-1}(I)} w(\mathcal{C}) .
$$

Corollary 5.3 The Coxeter fan refines the normal fan of the brick polytope. 


\subsection{Increasing flips and quotients of the weak order}

If $I$ and $J$ are adjacent facets of $\mathcal{S}(\mathrm{Q})$ with $I \backslash i=J \backslash j$, we say that the flip from $I$ to $J$ is increasing if $i<j$. This is equivalent to $\mathrm{r}(I, i) \in \Phi^{+}$(by Lemma 3.2 2) ), and to $f(\mathrm{~B}(I))<f(\mathrm{~B}(J))$, where $f$ denotes a linear function positive on $\Phi^{+}$(by Lemma 4.3). The graph of increasing flips is thus clearly acyclic, and we call increasing flip order its transitive closure $\prec$. Note that each face of the subword complex $\mathcal{S}(\mathrm{Q})$ has a unique minimal and a unique maximal element for $\prec$. This order generalizes the Tamari order on triangulations and is used by the first author in [Pil12] to construct a canonical spanning tree of the ridge graph of $\mathcal{S}(\mathrm{Q})$, obtaining a generating scheme for the facets of $\mathcal{S}(\mathrm{Q})$.

Proposition 5.4 A facet $I$ is covered by a facet $J$ in increasing flip order if and only if there exist $w_{I} \in \kappa^{-1}(I)$ and $w_{J} \in \kappa^{-1}(J)$ such that $w_{I}$ is covered by $w_{J}$ in weak order. We say that the increasing flip order is the quotient of the weak order by the map $\kappa$.

Proof: Let $w \in W$ and $s \in S$ such that $w<w s$ in weak order. Since $w\left(\alpha_{s}\right) \in \Phi^{+}$, Lemma 5.1 ensures that either $\kappa(w s)=\kappa(w)$ or $\kappa(w s)$ is obtained from $\kappa(w)$ by an increasing flip.

Reciprocally, consider two facets $I, J$ of $\mathcal{S}(\mathrm{Q})$ related by an increasing flip. We write $I \backslash i=J \backslash j$, with $i<j$. Their normal cones $\mathrm{C}^{\diamond}(I)$ and $\mathrm{C}^{\diamond}(J)$ are adjacent along a cone of codimension 1 which belongs to the hyperplane $H$ orthogonal to $r(I, i)=\mathrm{r}(J, j)$. Let $\mathcal{C}_{I}$ be a chamber of the Coxeter arrangement of $W$ which is contained in $C^{\diamond}(I)$ and adjacent to hyperplane $H$, and let $\mathcal{C}_{J}:=s_{\mathrm{r}(I, i)}\left(\mathcal{C}_{I}\right)$ denote its symmetric chamber with respect to $H$. Let $w_{I}$ and $w_{J}$ be such that $\mathcal{C}_{I}=w_{I}(\mathcal{C})$ and $\mathcal{C}_{J}=w_{J}(\mathcal{C})$. Then $w_{I} \in \kappa^{-1}(I)$ and $w_{J} \in \kappa^{-1}(J)$, and $w_{J}$ covers $w_{I}$ in weak order.

It follows from this proposition that $I \prec J$ in increasing flip order if there exists $w_{I} \in \kappa^{-1}(I)$ and $w_{J} \in \kappa^{-1}(J)$ such that $w_{I}<w_{J}$ in weak order. We conjecture that the converse also holds in general.

In his work on Cambrian lattices [Rea04, Rea06, Rea07], N. Reading considers quotients of the weak order under lattice congruences, which fulfill sufficient conditions for the quotient to be moreover a lattice. In the context of brick polytopes, we obtain different partitions of $W$ which are not lattice congruences in general (the fibers of $\kappa$ are not necessarily intervals of the weak order, but they contain all intervals between two of their elements). We nonetheless conjecture that the resulting quotient is in general a lattice, but this is still work in progress.

\subsection{Minkowski sum decomposition into Coxeter matroid polytopes}

Proposition 5.5 The brick polytope $\mathcal{B}(\mathrm{Q})$ is the Minkowski sum, over all positions $k \in[m]$, of the polytopes $\mathcal{B}(\mathrm{Q}, k):=\operatorname{conv}\{\mathrm{w}(I, k) \mid I$ facet of $\mathcal{S}(\mathrm{Q})\}$. In other words, the sum and convex hull commute:

$$
\mathcal{B}(\mathrm{Q}):=\operatorname{conv}_{I} \sum_{k} \mathrm{w}(I, k)=\sum_{k} \operatorname{conv}_{I} \mathrm{w}(I, k)=: \sum_{k} \mathcal{B}(\mathrm{Q}, k),
$$

where $k$ ranges over the positions $[m]$ and I ranges over the facets of the subword complex $\mathcal{S}(\mathrm{Q})$.

Proof: The proof is identical to that of [PS12a, Proposition 3.26].

Moreover, we observe that each summand $\mathcal{B}(\mathrm{Q}, k)$ is a $W$-matroid polytope as defined in [BGW97], i.e. a polytope such that the group generated by the mirror symmetries of its edges is a subgroup of $W$. 


\section{Cluster complexes and generalized associahedra revisited}

The cluster complex as defined in [FZ03] encodes the exchange graph of clusters in cluster algebras. Its first polytopal realization was obtained in [CFZ02]. Later on, an independent realization was presented in [HLT11], based on the $c$-Cambrian fans [Rea06, Rea07, RS09]. Recently S. Stella generalized in [Ste11] the approach of [CFZ02] and showed that the resulting realizations of the associahedra coincide with that of [HLT11]. In this section, we first study the brick polytope for the cluster complex as a spherical subword complex as defined in [CLS11]. In particular, we show that the polar of the brick polytope indeed realizes the cluster complex, and provide a description of its normal vectors and facets. As a consequence, we show that the brick polytope and the realization in [HLT11] differ only by an affine translation. This opens new perspectives on the latter realization, and provides new proofs of several constructions therein, in particular their connections with the Cambrian lattices and fans studied in [Rea06, Rea07, RS09]. Due to space limitation, we refer again to the long version of this paper [PS12b] for examples of 3-dimensional associahedra, with concrete computations of their vertices.

\subsection{Realizing cluster complexes}

Let $c$ be a Coxeter element, i.e. the product of all simple reflections in a given order. Following [Rea07], we choose an arbitrary reduced expression $\mathrm{c}$ of $c$ and let $\mathrm{w}_{\circ}(\mathrm{c})$ denote the c-sorting word of $w_{\circ}$, i.e. the lexicographically first (as a sequence of positions) reduced subword of $\mathrm{c}^{\infty}$ for $w_{\mathrm{o}}$. Extending properties in [PP12] and [Stu11] for type A subword complexes, it is proved in [CLS11] that the subword complex $\mathcal{S}\left(\mathrm{cw}_{\circ}(\mathrm{c})\right)$ is isomorphic to the cluster complex. Let $N:=\ell\left(w_{\circ}\right)=\left|\Phi^{+}\right|$, and $m:=n+N$ be the length of $\mathrm{cw}_{\circ}(\mathrm{c})$. The following theorem is now an easy consequence of the results developed in this paper.

Theorem 6.1 The polar of the brick polytope $\mathcal{B}\left(\mathrm{cw}_{\circ}(\mathrm{c})\right)$ realizes the subword complex $\mathcal{S}\left(\mathrm{cw}_{\circ}(\mathrm{c})\right)$.

Proof: The subset $[n]$ of positions of $[m]$ forms a facet of the $\mathcal{S}\left(\mathrm{cw}_{\circ}(\mathrm{c})\right)$ since its complement $\mathrm{w}_{\circ}(\mathrm{c})$ is a reduced expression for $w_{\circ}$. Its root configuration $\mathrm{R}([n])$ coincides with the root basis $\Delta$. Thus, the word $\mathrm{cw}_{\circ}(\mathrm{c})$ is realizing, and Theorem 4.6 ensures that the subword complex $\mathcal{S}\left(\mathrm{cw}_{\circ}(\mathrm{c})\right)$ is isomorphic to the boundary complex of the polar of the brick polytope $\mathcal{B}\left(\mathrm{cw}_{\circ}(\mathrm{c})\right)$.

\subsection{Normal vectors and facets}

Let $\mathrm{w}_{1}, \ldots, \mathrm{w}_{N}$ denote the letters of $\mathrm{w}_{\circ}(\mathrm{c})$. For $k \in[N]$, define $\rho_{k}:=w_{1} \cdots w_{k} \in W$ to be the product of the first $k$ letters of $\mathrm{w}_{\circ}(\mathrm{c})$ (with the convention $\rho_{0}:=e$ ). Moreover, let $\alpha_{k}:=\rho_{k-1}\left(\alpha_{s_{k}}\right), \omega_{k}:=\rho_{k-1}\left(\omega_{s_{k}}\right)$, and $\Omega:=\sum_{k \in[N]} \omega_{k}$. Remember that $\nabla:=\left\{\omega_{s} \mid s \in S\right\}$ denotes the set of fundamental weights.

Proposition 6.2 For any facet I of $\mathcal{S}\left(\mathrm{cw}_{\circ}(\mathrm{c})\right)$, and any position $i \in I$, the weight $\mathrm{w}(I, i)$ is a normal vector of the maximal face cone $\{\mathrm{r}(I, j) \mid j \in I \backslash i\}$ of the cone $\mathrm{C}(I)$. Thus, the normal vectors of the facets of $\mathcal{B}\left(\mathrm{cw}_{\circ}(\mathrm{c})\right)$ are given by $\nabla \cup\left\{\omega_{k} \mid k \in[N]\right\}$.

Proof: We derive this result from Proposition 3.9 and Lemma 5.2 in [CLS11]. The first proposition gives a rule to cyclically rotate the word $\mathrm{cw}_{\circ}(\mathrm{c})$ until the letter $i$ comes first, while maintaining the combinatorics of the subword complex. The second proposition ensures that, when $1 \in I$, the facet $I$ contains only letters $j$ for which $\mathrm{r}(I, j)$ belongs to $\Phi_{\left\langle\mathrm{c}_{1}\right\rangle}:=\Phi \cap \operatorname{vect}\left\{\alpha_{s} \mid s \in S \backslash \mathrm{c}_{1}\right\}$. Thus, $\mathrm{w}(I, 1)$ is orthogonal to all the roots of $\mathrm{R}(I) \backslash \mathrm{r}(I, 1)$. This proves the first sentence of the proposition. The result follows since the weight $\mathrm{w}(I, i)$ is by definition of the form $\omega_{s}$, for some $s \in S$, or $\omega_{k}$, for some $k \in[N]$. 
To obtain the inequality description of the brick polytope $\mathcal{B}\left(\mathrm{cw}_{\circ}(\mathrm{c})\right)$, we now need to study carefully the facets $\kappa\left(w_{k}\right)$ of $\mathcal{S}\left(\mathrm{cw}_{\circ}(\mathrm{c})\right)$. The following lemma gives their root configurations and brick vectors. We skip its proof since it is technical and not enlightening. See [PS12b] for details.

Lemma 6.3 For any $k \in\{0, \ldots, N\}$, we have $\mathrm{R}\left(\kappa\left(\rho_{k}\right)\right)=\rho_{k}(\Delta)$ and $\mathrm{B}\left(\kappa\left(\rho_{k}\right)\right)=\Omega+\rho_{k}\left(\sum_{s \in S} \omega_{s}\right)$.

Let $q:=\sum_{s \in S} \omega_{s}$ and let $\mathcal{P}(W):=\operatorname{conv}\{w(q) \mid w \in W\}$ denote the balanced $W$-permutahedron.

Theorem 6.4 The brick polytope $\mathcal{B}\left(\mathrm{cw}_{\circ}(\mathrm{c})\right)$ is obtained from the balanced permutahedron $\mathcal{P}(W)$ removing all facets which do not intersect $\left\{\rho_{k}(q) \mid k \in[N]\right\}$, and then translating by the vector $\Omega:=\sum_{k \in[N]} \omega_{k}$.

Proof: Let $\mathcal{B}^{\prime}$ denote the translate of $\mathcal{B}\left(\mathrm{cw}_{\circ}(\mathrm{c})\right)$ by the vector $-\Omega$. According to Lemma 6.3 , the vertices of $\mathcal{B}^{\prime}$ and $\mathcal{P}(W)$ corresponding to the prefixes $w_{p}$ of $\mathrm{w}_{\circ}(\mathrm{c})$ coincide, as well as their normal cones. Thus, the facets of $\mathcal{B}^{\prime}$ and $\mathcal{P}(W)$ containing these vertices are defined by the same inequalities. The result thus follows from Proposition 6.2 which affirms that these inequalities exhaust all facets of $\mathcal{B}^{\prime}$.

Corollary 6.5 Up to the translation by the vector $\Omega$, the brick polytope $\mathcal{B}\left(\mathrm{cw}_{\circ}(\mathrm{c})\right)$ coincides with the c-associahedron $\operatorname{Asso}_{c}^{q}(W)$ in [HLT11].

We thus obtained in the present paper the vertex description of these polytopes (by Definition 4.1), as well as a natural Minkowski sum decomposition into $W$-matroid polytopes (by Proposition 5.5). In the end of this paper, we revisit various results contained in [HLT11]. The approach developed in this paper, based on subword complexes and brick polytopes, enables us to simplify certain proofs and connections. First, we provide a new way to see that the $c$-singletons as defined in [HLT11] can be used to characterize the vertices common to the brick polytope $\mathcal{B}\left(\mathrm{cw}_{\circ}(\mathrm{c})\right)$ and to the balanced $W$-permutahedron $\mathcal{P}(W)$. We omit again the proof which can be found in [PS12b].

Proposition 6.6 The following properties are equivalent for an element $w \in W$ :

(i) The element $w$ is a singleton for the map $\kappa$, i.e. $\kappa^{-1}(\kappa(w))=\{w\}$.

(ii) The root configuration of $\kappa(w)$ is given by $\mathrm{R}(\kappa(w))=w(\Delta)$.

(iii) The weight configuration $\mathrm{W}(\kappa(w))$ of $\kappa(w)$ is given by $\mathrm{W}(\kappa(w))=w(\nabla)$.

(iv) The vertices $\mathrm{B}(\kappa(w))$ of the brick polytope $\mathcal{B}\left(\mathrm{cw}_{\circ}(\mathrm{c})\right)$ and $w(q)$ of the balanced $W$-permutahedron $\mathcal{P}(W)$ coincide up to $\Omega$, i.e. $\mathrm{B}(\kappa(w))=\Omega+w(q)$.

(v) There exist reduced expressions $\mathrm{w}$ of $w$ and $\mathrm{c}$ of $c$ such that $\mathrm{w}$ is a prefix of $\mathrm{w}_{\circ}(\mathrm{c})$.

(vi) The complement of $\kappa(w)$ in $\mathrm{cw}_{\circ}(\mathrm{c})$ is $\mathrm{w}_{\circ}(\mathrm{c})$.

\subsection{Cambrian lattices and fans}

N. Reading inductively defines the set $\operatorname{Sort}_{c}(W)$ of $c$-sortable elements as follows. Let $w \in W$, and let $s$ be initial in $c$, i.e. $\ell(s c)<\ell(c)$. Then

$$
w \in \operatorname{Sort}_{c}(W) \Longleftrightarrow \begin{cases}s w \in \operatorname{Sort}_{s c s}(W) & \text { if } \ell(s w)<\ell(w) \\ w \in \operatorname{Sort}_{s c}\left(W_{\langle s\rangle}\right) & \text { if } \ell(s w)>\ell(w)\end{cases}
$$


The first case yields an induction on the length $\ell$, while the second case is an induction on the rank of $W$. The downward projection which associates to any element $w \in W$ the maximal $c$-sortable element below $w$ is denoted by $\pi_{\downarrow}^{c}$. We now describe the analogue inductive nature of the facets of $\mathcal{S}\left(\mathrm{cw}_{\circ}(\mathrm{c})\right)$. To this end, we define the rotated word of $\mathrm{Q}:=\mathrm{q}_{1} \mathrm{q}_{2} \cdots \mathrm{q}_{m}$ to be $\mathrm{Q}_{\circlearrowleft}:=\mathrm{q}_{2} \cdots \mathrm{q}_{m} \psi\left(q_{1}\right)$, where $\psi$ denotes the automorphism on $S$ defined by $\psi(s):=w_{\circ}^{-1} s w_{\circ}$. Let $I$ be a facet of $\mathcal{S}\left(\mathrm{cw}_{\circ}(\mathrm{c})\right)$, and let $s$ be initial in $c$. Then there are two cases.

(i) The first occurrence of $s$ in $\mathrm{cw}_{\circ}(\mathrm{c})$ is not contained in $I$. We then have by [CLS11, Proposition 4.3] that $I$, shifted by one, is a facet of the subword complex for $\left(\mathrm{cw}_{\circ}(c)\right)_{\circlearrowleft}$, which is, up to commutations of consecutive commuting letters, equal to $\mathrm{c}^{\prime} \mathrm{w}_{\circ}\left(c^{\prime}\right)$ with $c^{\prime}:=s c s$.

(ii) The first occurence of $s$ in $\mathrm{cw}_{\circ}$ (c) is contained in $I$. [CLS11, Lemma 5.2] then ensures that $k \notin I$ for all $k$ such that $\mathrm{r}(I, k) \in \Phi \backslash \Phi_{\langle s\rangle}$. By [CLS11, Lemma 5.4], we therefore obtain that $I$ can also be considered as a facet of the subword complex for the word $\mathrm{c}^{\prime} \mathrm{w}_{\circ}\left(c^{\prime}\right)$ with $c^{\prime}:=s c$.

This analogous inductive nature enables us to connect the increasing flip order with the Cambrian lattice and the normal fan of the brick polytope with the Cambrian fan.

Theorem 6.7 Let $v, w \in W$ and let $v$ be c-sortable. Then $\pi_{\downarrow}^{c}(w)=v \Longleftrightarrow \kappa(w)=\kappa(v)$.

Corollary 6.8 The Cambrian lattice on c-sortable elements in $W$ and the increasing flip order on the facets of $\mathcal{S}\left(\mathrm{cw}_{\circ}(\mathrm{c})\right)$ are isomorphic.

Corollary 6.9 The normal fan of the brick polytope $\mathcal{B}\left(\mathrm{cw}_{\circ}(\mathrm{c})\right)$ coincides with the c-Cambrian fan.

The construction of the $c$-associahedron of [HLT11] is based on the $c$-Cambrian fan. Corollary 6.9 could thus be deduced from the connection between the brick polytope $\mathcal{B}\left(\mathrm{cw}_{\circ}(\mathrm{c})\right)$ and the $c$-associahedron of [HLT11]. However, the brick polytope approach provides a new independent proof of this result.

\section{Acknowledgements}

The second author wants to thank Pavlo Pylyavskyy for pointing him to [BZ97] which was the starting point for the present paper. We thank Cesar Ceballos, Frédéric Chapoton, Christophe Hohlweg, JeanPhilippe Labbé, Carsten Lange, Nathan Reading, and Hugh Thomas for fruitful discussions on the topic. The first author also thanks Francisco Santos and Michel Pocchiola for their inspiration and collaboration on the premises of brick polytopes in type $A$. He is also grateful for the opportunity to visit the LaCIM at UQÀM, where this work started. He finally acknowledges the Fields Institute of Toronto for his postdoctoral stay in Canada, and Antoine Deza for his supervision.

\section{References}

[BGW97] Alexandre V. Borovik, Israel M. Gelfand, and Neil White. Coxeter matroid polytopes. Ann. Comb., 1(2):123-134, 1997.

[BZ97] Arkady Berenstein and Andrei Zelevinsky. Total positivity in Schubert varieties. Comment. Math. Helv., 72(1):128-166, 1997.

[CFZ02] Frédéric Chapoton, Sergey Fomin, and Andrei Zelevinsky. Polytopal realizations of generalized associahedra. Canad. Math. Bull., 45(4):537-566, 2002. Dedicated to Robert V. Moody. 
[CLS11] Cesar Ceballos, Jean-Philippe Labbé, and Christian Stump. Subword complexes, cluster complexes, and generalized multi-associahedra. Preprint, arXiv:1108.1776, 2011.

[FZ03] Sergey Fomin and Andrei Zelevinsky. Cluster algebras. II. Finite type classification. Invent. Math., 154(1):63-121, 2003.

[HL07] Christophe Hohlweg and Carsten E. M. C. Lange. Realizations of the associahedron and cyclohedron. Discrete Comput. Geom., 37(4):517-543, 2007.

[HLT11] Christophe Hohlweg, Carsten E. M. C. Lange, and Hugh Thomas. Permutahedra and generalized associahedra. Adv. Math., 226(1):608-640, 2011.

[Hum90] James E. Humphreys. Reflection groups and Coxeter groups, volume 29 of Cambridge Studies in Advanced Mathematics. Cambridge University Press, Cambridge, 1990.

[KM04] Allen Knutson and Ezra Miller. Subword complexes in Coxeter groups. Adv. Math., 184(1):161-176, 2004.

[KM05] Allen Knutson and Ezra Miller. Gröbner geometry of Schubert polynomials. Ann. of Math. (2), 161(3):1245-1318, 2005.

[Pil12] Vincent Pilaud. Greedy flip trees in subword complexes. Preprint, arXiv:1203.2323, 2012.

[PP12] Vincent Pilaud and Michel Pocchiola. Multitriangulations, pseudotriangulations and primitive sorting networks. Discrete Comput. Geom. (DOI: 10.1007/s00454-012-9408-6), 2012.

[PS12a] Vincent Pilaud and Francisco Santos. The brick polytope of a sorting network. European J. Combin., 33(4):632-662, 2012.

[PS12b] Vincent Pilaud and Christian Stump. Brick polytopes of spherical subword complexes: a new approach to generalized associahedra. Preprint, arXiv:1111.3349, 2012.

[Rea04] Nathan Reading. Lattice congruences of the weak order. Order, 21(4):315-344 (2005), 2004.

[Rea06] Nathan Reading. Cambrian lattices. Adv. Math., 205(2):313-353, 2006.

[Rea07] Nathan Reading. Sortable elements and Cambrian lattices. Algebra Universalis, 56(3-4):411437, 2007.

[RS09] Nathan Reading and David E. Speyer. Cambrian fans. J. Eur. Math. Soc. (JEMS), 11(2):407447, 2009.

[SS12] Luis Serrano and Christian Stump. Maximal fillings of moon polyominoes, simplicial complexes, and schubert polynomials. Electron. J. Combin., 19, 2012.

[Ste11] Salvatore Stella. Polyhedral models for generalized associahedra via coxeter elements. Preprint, arXiv:1111.1657, 2011.

[Stu11] Christian Stump. A new perspective on $k$-triangulations. J. Combin. Theory Ser. A, 118(6):1794-1800, 2011. 